\title{
DIFERENCIAS EN BURNOUT SEGÚN SEXO Y EDAD EN PROFESORADO NO UNIVERSITARIO
}

\author{
Lucía Granados Alós \\ Universidad Internacional de Valencia, España \\ lucia.granados@campusviu.es \\ Ricardo Sanmartín López \\ Universidad de Alicante, España. \\ Nelly G. Lagos San Martín \\ Universidad del Bío-Bío, Chile. \\ María E. Urrea-Solano \\ Universidad de Alicante, España. \\ María J. Hernández-Amorós \\ Universidad de Alicante, España.
}

https://doi.org/10.17060/ijodaep.2017.n1.v2.945

Fecha de Recepción: 9 Febrero 2017

Fecha de Admisión: 1 Abril 2017

\section{RESUMEN}

El modelo de burnout más utilizado en los estudios sobre el profesorado, es el modelo trifactorial de Maslach, que describe tres tipos de síntomas, agotamiento emocional que conlleva cansancio físico y mental, despersonalización con sentimientos negativos, y sensación de bajo logro profesional que se traduce al pensar que las demandas superan los recursos para ser atendidas eficazmente. Como consecuencia del mismo se produce, en quienes lo padecen, un desánimo generalizado. El objetivo del presente estudio fue analizar las diferencias en burnout según el sexo y la edad. Para ello se reclutó una muestra 507 participantes, que impartían docencia en Educación Infantil, Educación Primaria y Educación Secundaria. Los participantes cumplimentaron el Maslasch Burnout Inventory. Los resultados de los análisis de varianza concretaron diferencias estadísticamente significativas tanto en función del sexo como de los grupos de edad. Estos hallazgos muestran la importancia de implementar programas orientados a la prevención de los síntomas.

Palabras clave: burnout, profesorado, agotamiento emocional, realización personal, despersonalización.

Lee (1990) destacó que las mujeres sufren mayor y más intenso cansancio emocional que los hombres; sin embargo, Gil-Monte y Silla, (1997), Schwab y Iwanicki, (1982) vieron que existía 
mayor varianza en despersonalización, siendo los hombres los que tienen un sentimiento más negativo hacia los alumnos. Dichos resultados se contradicen con los de Maslach y Jackson (1981), que encuentran justamente lo contrario, es decir, ellos establecen diferencias en un mayor cansancio emocional y menor realización personal en las mujeres. Otras autoras (Abraham, 1986; Maslach, 1982), centran su atención en el doble rol ejercido por la mujer en el trabajo y en la casa como agente potenciador del estrés. Pese a todo, siguen siendo una mayoría los autores que no encuentran una clara evidencia respecto a la variable sexo (Byrne, 1999; Schwab y Iwanicki, 1982).

En cuanto a la edad, los números estudios también muestran resultados contradictorios puesto que la relación que se establece entre la edad y el burnout ha sido directa para unos como Cabrera y Elvira, (2004) y no tanto para otros como Carlotto, (2008), Moriana y Herruzo,(2006), Whitehead y Lindquist, (1986) habiéndose encontrado también ausencia total de relación (Hock, 1988). Estos resultados se deberían de haber matizado porque la relación que se establece entre el síndrome de burnout y la edad, tiene que ver más con los años de experiencia en la profesión y la maduración del individuo por la edad. Por ejemplo, se presentan evidencias claras, que los profesores más jóvenes experimentan niveles mayores de estrés (Briones, Tabernero y Arenas, 2010) y también de cansancio emocional y fatiga (Crane y Iwanicki, 1986; Schwab y Iwanicki, 1982). Por otro lado, Borg y Falzon, (1990), Van Horn, Schaufeli, Greenglass y Burke, (1997) informaban que los docentes más experimentados, que llevaban más de veinte años en la profesión, tenían una respuesta de estrés mayor que la de sus compañeros (Moreira Lema, 2012 ). Mientras que Malik, Mueller y Meinke (1991), no informaban sobre diferencias significativas respecto a la edad.

Como consecuencia de lo anteriormente expuesto, en nuestro estudio se espera que existan diferencias estadísticamente significativas en las dimensiones de burnout en función del sexo y los grupos de edad de los participantes.

\section{PARTICIPANTES}

Nuestra muestra estuvo compuesta por 507 profesores (53,4\% mujeres) pertenecientes a centros concertados de las provincias de Alicante, Murcia y Valencia.

\section{MEDIDAS}

Maslach Burnout Inventory (MBI) (Maslach y Jackson, 1981).

Es el instrumento más utilizado para evaluar el nivel de Burnout, es un cuestionario autoadministrado, y está constituido por 22 ítems en forma de afirmaciones sobre los sentimientos y actitudes del profesional en su trabajo. Los valores del Alpha de Crombach de las subescalas originales son de .90 para el Agotamiento Emocional, .79 para Despersonalización y .71 para Realización Personal (Maslach y Jackson, 1982).

Agotamiento Emocional. Entendido como la disminución y pérdida de recursos emocionales, sensaciones de sobreesfuerzo físico y hastío emocional que se produce como consecuencia de las continuas interacciones de los profesores en su contexto de trabajo. Esta subescala consta de 9 ítems, con enunciados como "Me siento agotado/a emocionalmente a causa de mi trabajo" "Trabajar con personas me causa demasiada tensión" etc.

Despersonalización. Desarrollo de actitudes negativas, de insensibilidad y cinismo hacia los compañeros, padres, alumnos, etc. Consta de 5 ítems "Pienso que me he hecho más insensible con la gente", "Realmente no me preocupa lo que les pueda pasar a mis alumnos" etc.

Baja Realización Personal. Conlleva la pérdida de confianza en los logros propios en el contexto docente y la presencia de un negativo auto concepto. Implica sentimientos de incompetencia profesional, evalúa sentimientos de competencia y bienestar relativos al trabajo (ejemplo. "en este 
empleo consigo muchas cosas que valen la pena"). Esta subes cala está compuesta por 8 ítems como; "Me siento animado después de trabajar con mis alumnos".

En este estudio, las distintas subescalas del inventario demostraron su adecuada fiabilidad con valores de alpha de Cronbach igual a .86 (agotamiento emocional); .71 (despersonalización); y .74 (realización personal).

\section{PROCEDIMIENTO.}

En primer lugar se solicitó la autorización a los equipos directivos de los centros, posteriormente, se elaboró una carta informativa dirigida a los de los centros seleccionados, en la que se explicaba el propósito del estudio y se solicitaba su colaboración a participar en la investigación. Una vez concretados todos estos aspectos, se procedió al pase del cuestionario que fue administrado on line, y su cumplimentación fue voluntaria.

\section{ANÁLISIS ESTADÍSTICOS}

Para analizar el burnout en función del sexo y los grupos de edad se realizaron análisis de varianza (ANOVA). A su vez, se efectuaron análisis post hoc a través de la prueba Scheffé para identificar entre qué profesores se encontraban diferencias significativas.

\section{RESULTADOS}

La tabla 1 ofrece las medias y diferencias estadísticamente significativas halladas en función del sexo en base a las tres dimensiones estudiadas por Maslach Agotamiento Emocional (AE), Realización Personal (RP) y Despersonalización (D).

Las diferencias halladas entre los dos grupos resultaron estadísticamente significativas para las dimensiones de Agotamiento Emocional $(\mathrm{AE})\left(F_{(1,505)}=13.82 ; p<.001\right)$ y Realización Personal $(\mathrm{RP})\left(F_{(1,505)}=7.04 ; p=.008\right)$. El profesorado de sexo masculino obtuvo puntuaciones más altas que sus iguales de sexo femenino. Por el contrario, las profesoras obtuvieron puntuaciones más altas en Realización Personal $(\mathrm{RP})\left(F_{(1,505)}=7.8 ; p=.008\right)$, que los profesores. Tan sólo en una de las dimensiones, Despersonalización $D^{\prime}\left(F_{(1,505)}=.04 ; p=.86\right)$, no se obtuvieron diferencias significativas en función del sexo.

\section{Tabla 1}

Medias, desviación típica y significación estadística en las dimensiones del Burnout según el sexo.

\begin{tabular}{|c|c|c|c|c|c|c|}
\hline & \multicolumn{2}{|c|}{ Profesores } & \multicolumn{2}{|c|}{ Profesoras } & \multicolumn{2}{|c|}{$\begin{array}{c}\text { Significación } \\
\text { estadística }\end{array}$} \\
\hline & $M$ & $D E$ & $M$ & $D E$ & $F$ & $p$ \\
\hline $\mathbf{A E}$ & 1.16 & .39 & 1.16 & .47 & 13.82 & $<.001$ \\
\hline $\mathbf{R P}$ & 2.38 & .45 & 2.60 & .25 & 7.04 & .008 \\
\hline D & 1.62 & .31 & 1.38 & .25 & .04 & .865 \\
\hline
\end{tabular}

Despersonalización. 


\section{DIFERENCIAS EN BURNOUT SEGÚN SEXO Y EDAD EN PROFESORADO NO UNIVERSITARIO}

La tabla 2 ofrece las medias y diferencias estadísticamente significativas halladas en función de la edad en base a las tres dimensiones estudiadas por Maslach agotamiento emocional (AE), realización personal (RP) y despersonalización (D).

Las diferencias halladas entre los grupos resultaron significativas para las dimensiones de Agotamiento Emocional $(A E) F(3,503)=3.62 ; p=.014)$, Realización Personal $(R P)(F(3,503)=9.34$; $p<.001)$ y Despersonalización $(D)(F(3,503)=7.49 ; p<.001)$.

En la dimensión (Agotamiento Emocional) $(A E)$, las diferencias significativas reportaron que los profesores con un rango de edad entre los 30-39 y 40-49 años presentaron mayores puntuaciones en agotamiento emocional 30-39 $(M=1.15 ; D E=.45), 40-49(M=1.12 ; D E=.44)$ que sus iguales con edades comprendidas entre los $20-29$ años $(M=1.04 ; D E=.37) 0$ más de 50 años $(M=1.03$; $D E=.43)$.

En la dimensión Realización Personal $(R P)$, las puntuaciones evidenciaron que los profesores que mayor puntuación obtuvieron en realización personal fueron los de edades comprendidas entre los 20-29 años $(M=2.64 ; D E=.30)$, en comparación con sus iguales en las que las puntuaciones fueron 30-39 ( $M=1.15 ; D E=.45), 40-49(M=1.12 ; D E=.44) 0<50(M=1.03 ; D E=.43)$.

En la dimensión Despersonalización (D), al analizar la media, para determinar el nivel de despersonalización del burnout en función de la edad, los resultados reportan, cómo los profesores más jóvenes presentan niveles de Despersonalización mayores, 20-29 años $(M=1.65$; $D E=.35)$, y 3039 años $(M=1.65 ; D E=.42)$, que los grupos de mayor edad $40-49(M=1.58 ; D E=.32)$ y de más de 50 años $(M=1.49 ; D E=.27)$.

Tabla 2

Medias, desviación típica y significación estadística de las dimensiones del Burnout según la edad.

20-29 años 30-39 años 40-49 años 50 o mayor Significación

estadística

\begin{tabular}{lllllllllll}
\hline & $M$ & $D E$ & $M$ & $D E$ & $M$ & $D E$ & $M$ & $D E$ & $F$ & $p$ \\
\hline AE & 1.04 & .37 & 1.15 & .45 & 1.12 & .44 & 1.03 & .43 & 3.62 & .014 \\
RP & 2.64 & .30 & 2.52 & .45 & 2.39 & .51 & 2.59 & .37 & 9.34 & $<.001$ \\
& & & & & & & & & & \\
D & 1.65 & .35 & 1.65 & .42 & 1.58 & .32 & 1.49 & .27 & 7.49 & $<.001$
\end{tabular}

Nota. $\mathrm{AE}=$ Agotamiento emocional; $\mathrm{RP}=$ Realización personal; $\mathrm{D}=$

Despersonalización.

\section{DISCUSIÓN Y CONCLUSIONES.}

En nuestro estudio, se hallaron diferencias significativas en agotamiento emocional y en realización personal y no en despersonalización. Los profesores puntuaron más en AE y ellas más en RP. Estos datos coinciden con los aportados por Maslach y Jackson, (1981). Otros estudios consultados difieren del nuestro al manifestar que, en la relación entre el Burnout y el sexo se encontraron diferencias significativas en todas las dimensiones ( $A E, R P$ y $D$ ), y además difieren también en que 
fueron las profesoras las que evidenciaron mayores niveles de Agotamiento Emocional (Araújo, 2008; García-Renedo et al., 2004; Lee 1990; Marente y Gestoso, 2008; López-Morales et al., 2006; Rubino et al., 2009; Salanova et al., 2003; Van den Broeck, et al., 2008), menores niveles de Realización Profesional (Masclach y Jackson, 1981; López-Morales et al., 2006) y menores niveles de Despersonalización (Masclach y Jackson, 1981; López-Morales et al., 2006; Macías et al., 2007; Viloria-Marín, y Paredes-Santiago, 2002), lo que deja ver una mayor tendencia de los hombres para el distanciamiento profesional y emocional relativamente a los alumnos, acabando por volverse menos sensibles a las problemáticas que estos presentan y a la ayuda efectiva de sus dificultades escolares y personales, utilizando este cinismo como forma de coping (Schaufeli y Ezmann, 1998). Otras autoras (Abraham, 1986; Maslach, 1982), centran su atención en el doble rol ejercido por la mujer en el trabajo y en la casa como agente potenciador del estrés.

Byrne (1999) y Schwab y Iwanicki (1982), no hallaron diferencias significativas en burnout según el sexo. Estos resultados no coinciden con los del estudio de Cardoso, y Grilo (2002), en términos de significación estadística ni con nuestra investigación ya que como hemos comentado con anterioridad, ellas presentaban menores niveles de agotamiento emocional, mayores niveles de realización personal e igual nivel de despersonalización que sus compañeros de sexo masculino.

En cuanto a la edad en nuestro estudio se hallaron diferencias significativas en AE, RP y D coincidiendo con los publicados por Cabrera y Elvira, (2004); Gomes et al. (2006) y Mota-Cardoso et al. (2002), en los que también se hallaron diferencias estadísticamente significativas, pero difieren de los resultados obtenidos en los estudios de (Moriana y Herruzo, 2006; Carlotto, 2008 ; Hock, 1988;Whitehead y Lindquist, 1986) en los que se encuentra ausencia total de relación.

Nuestro estudio corrobora parte de la investigación realizada por Briones, Tabernero y Arenas (2010) sin embargo, en relación con los estudios encontrados, fueron los profesores con edades comprendidas entre los 30 y 59 años los más agotados emocionalmente (Salanova et. al., 2003), con menos realización profesional en el desempeño de la labor docente y con menores niveles de despersonalización (Correia, et al., 2010; García-Renedo et al. 2004). Por tanto, no se corresponde con nuestro estudio, en el que hemos visto que los más agotados emocionalmente son los de edades intermedias 30-39, los más realizados personalmente los más jóvenes 20-29 y los más mayores más de 50, con mayor nivel de despersonalización.

\section{REFERENCIAS BIBLIOGRÁFICAS}

Abraham, A. (1986). El enseñante es también una persona. Barcelona: Gedisa.

Araújo, A. M. (2008). Stress nos docentes portugueses: relação com o exercício, com o Burnout, com a saúde e com auto-eficácia geral segundo (Tesis Doctoral). Universidad Miguel Hernandez, Alicante, España.

Borg, M.G., y Falzon, J.M., (1990). Teachers'perception of primary schoolchildren's undesirable behaviours. the effects of teaching experience, pupil's age, sex and ability stream. British Journal of Educational Psychology, 60(2), 220-226.

Briones, E., Tabernero, C., y Arenas, A. (2010). Satisfacción Laboral de los Profesores de Secundaria: Efecto de Factores Demográficos y Psicosociales. Revista de Psicología del Trabajo $y$ de las Organizaciones, 26(2), 115-122.

Byrne, B., (1999). The nomological network of teacher burnout: A literature review and empirically validated model. Nueva York. Cambridge University Press.

Cabrera, J. H., y Elvira, J. A. (2004). Estrés y burnout en profesores. International journal of clinical and health psychology, 4(3), 597-621.

Cardoso, R. M., y Grilo, M. (2002). O stress nos professores portugueses: Estudo IPSSO 2000. 
Carlotto, M. S. (2008). Análise da produção científica sobre a Síndrome de Burnout no Brasil. Psico, 39(2), 152-158.

Correia, T., Gomes, A. R., y Moreira, S.. (2010). Stresse ocupacional em professores do ensino básico: Um estudo sobre as diferenças pessoais e profissionais. 10(1), 1477-1493.

Crane, S. J., y Iwanicki, E. F. (1986). Perceived role conflict, role ambiguity, and burnout among special education teachers. Remedial and Special Education, 7(2), 24-31.

García-Renedo, M., Salanova, S., y Cifre, E. (2004). Antecedentes afectivos de la autoeficacia entre profesores: diferencias individuales. Nuevos horizontes en la investigación sobre la autoeficacia. Castelló de la Plana. Publicacions de la Universitat Jaume I.

Gil-Monte, P. R., y Silla, J. M. P. (1997). Desgaste psíquico en el trabajo: el síndrome de quemarse. Madrid: Síntesis.

Hock, R. R. (1988). Professional burnout among public school teachers. Public personnel management. 17(2), 167-189.

Lee, R. T. (1990). On the meaning of Maslach's three dimensions of burnout. Journal of applied psychology, 75(6), 743. Doi. 10.1037/0021-9010.75.6.743

López-Morales, A., González-Velázquez, F., Morales-Guzmán, M. I., y Espinoza-Martínez, C. E. (2006). [The burnout syndrome in medical residents working long periods]. Revista médica del Instituto Mexicano del Seguro Social, 45(3), 233-242.

Macías, A. B., Soto, R. C., y Corral, M. G. (2007). Síndrome de Burnout: Un estudio comparativo entre profesores y médicos de la ciudad de Durango. Investigación Educativa Duranguense, (6), 64-73.

Malik J.L., Mueller R.0.,y Meinke D.L. (1991). The effects of teaching experience and grade level taught on teacher stress: A LISREL analysis. Teaching and Teacher Education, 7(1), 57-62. Doi. 10.1016/0742-051X (91)90057-V

Marente, J. A. A., y Gestoso, C. L. G. (2008). Burnout y mobbing en enseñanza secundaria. Revista Complutense de Educación, 19(1), 157.

Maslach C., y Jackson, S.E., (1982.). Burnout in health professions. A social psychological analysis. In G. Sanders e J. Suls (Eds.), Social Psychology of health and illness, 2, 227-251. Doi. 10.1007/BF00287876.

Maslach, C. (1982). Burnout. The Cost of Caring. Nueva York: Prentice-Hall Press.

Moreira Lema, S. L. (2012). Identificación del burnout en profesionales de la salud y factores relacionados con este fenómeno, en la ciudad de Santo Domingo de los Tsáchilas, hospital Dr. Gustavo Domínguez de Santo Domingo de los Tsáchila.

Moriana, J.A. y Herruzo, J (2006). Variables related to psychiatric sick leave taken by Spanish secondary school teachers. Work y Stress, 20(3), 259-271.

Rubino, C., Luksyte, A., Perry, S. J., y Volpone, S. D. (2009). How do stressors lead to burnout? The mediating role of motivation. Journal of Occupational Health Psychology, 14(3), 289. Doi. $10.1037 / \mathrm{a} 0015284$.

Salanova, M., Llorens, S., y García-Renedo, M. (2003). ¿Por qué se están quemando los profesores? Prevención, trabajo y salud, 28, 16-20.

Schwab, R.L., y Iwanicki, E.F., (1982). Perceived role conflict, role ambiguity, and teacher burnout. Educational Administration Quarterly, 18(1),60-74. Doi. 10.1177/ $0013161 \times 82018001005$

Van den Broeck, A., Vansteenkiste, M., De Witte, H., y Lens, W. (2008). Explaining the relationships between job characteristics, burnout, and engagement: The role of basic psychological need satisfaction. Work \& Stress, 22(3), 277-294. Doi. 10.1080/02678370802393672. 
Viloria-Marín, H., y Paredes-Santiago, M. (2002). Estudio del síndrome de burnout o desgaste profesional en los profesores de la Universidad de los Andes. Educere, 6(17), 29-36.

Whitehead,J.T, y Lindquist,C.A., (1986). Correctional officer job burnout: A path model. Journal of Research in Crime and Delinquency, 23(1), 23-42. Doi. 10.1177/0022427886023001003

Winzelberg, A. J., y Luskin, F. M. (1999). The effect of a meditation training in stress levels in secondary school teachers. Stress and Health, 15(2), 69-77. 
